\title{
Effect of Açaí Powder and Polydextrose on Bread Quality Using Surface Response
}

Rafael Audino Zambelli, Antonio Weskley Soares, Luan Icaro Freitas Pinto, Cícera Alyne Lemos Melo, Luciana Gama de Mendonça, Ana Caroline da Silva, Glauber Batista Moreira Santos, Marina Lisboa Silva and Maryana Monteiro Farias

Food Engineering Department, Federal University of Ceará, 2977Mister Hull Avenue, Fortaleza 60356001, Brazil

\begin{abstract}
The aim of this study was to develop a new bread formulation from different amounts of açaí powder and polydextrose incorporated into wheat flour on bread technological properties. The formulations were developed using the central composite rotational design and having as dependent variables specific volume, hardness, crumb color and image analysis. Statistical analysis was performed using multiple regression and response surface. The results showed that the inclusion of powdered açaí and polydextrose in bread formulations reduces the specific volume and alveoli number and increases the browning and hardness of the bread. However, values of this addition $(30 \mathrm{~g} / 100 \mathrm{~g})$ were found that did not promote significant quality reduction and the scanning electron microscopy (SEM) showed minimal damage to the gluten network at this level of incorporation.
\end{abstract}

Key words: Bread quality, fermentation, functional ingredients, gluten network.

\section{Introduction}

In today's world, one of the major nutritional problems is the consumption of high quantities of fat and sugar, which has been associated with serious health problems. Due to a growing public interest in such products, the food industry has focused on developing low-calorie foods. The key means applied to reduce the energetic value of bakery products (including short-dough biscuits) include the use of whole-grain flours, sweeteners for partial replacement of sugar (e.g. tagatose, trehalose, inulin, oligofructose, polydextrose) [1].

A high intake of fruit and vegetables reduces the risk of age related disease [2]. Mechanistic studies suggest that these effects may be mediated, in part, by the interaction of undigested plant foods, and not limited to the traditional definitions of dietary fiber, with the gut microbiota [3].

Açai (Euterpe oleracea Martius) tree is a large

Corresponding author: Rafael Audino Zambelli, adjunct professor, research fields: baking and starch technology, food processing and food engineering. palm found in the Amazon flood plain. The fruit of this palm is a small purple-black berry which reaches about $10 \mathrm{~mm}$ in diameter and is usually consumed in all states of Brazil since it is rich in $\alpha$-tocopherol, fibers, lipids, polyphenols (including anthocyanins), and mineral ions [4], has received much attention in recent years due to its potential anti-oxidant and anti-inflammatory properties [5].

Polydextrose (PDX) is a soluble fiber with low viscosity that provides one-fourth of the energy that is supplied by glucose [6] and consists of approximately $89 \%$ of d-glucose, $10 \%$ of sorbitol and $1 \%$ of citric acid [7], easily dissolves in water and, owing to its technological traits, may be used as a replacer of both fat and sugar [8]. Recent studies suggest that its consumption governs appetite. In a meta-analysis, we found that PDX reduces subsequent energy intake (EI) dose-dependently [9] and alters subjective feelings of appetite [10]. It has been successfully incorporated into a wide range of foods, i.e. baked goods, confectionery, beverages as well as frozen desserts, while providing the appropriate textural and mouthfeel 
properties [11].

The inclusion of dietary fiber into food matrix (e.g. bread, cake, donut, etc.) could improve the daily intake value, thereby decrease the risks of constipation, diverticulosis, colon cancer, gallstone formation, overweight, hypercholesterolemia, type 2 diabetes, and atherosclerosis [12-14].

Bread, the product of wheat flour fermentation by yeast, is widely consumed as a staple food across many cultures and countries worldwide [15]. The functionality of the gluten network developed through mixing is crucial for gas retention and the final structure of bread. A previous study demonstrated that the impact of vacuum mixing on gluten network development was flour-type dependent [16]. The inclusion of açaí powder and polydextrose can improve the nutritional value of breads; however, the damage to gluten network for these raw materials is still unknown.

The aim of this study was to develop a new bread formulation from different amounts of açaí powder and polydextrose incorporated into wheat flour. The effects of açaí powder and polydextrose were evaluated by means of technological properties of bread.

\section{Materials and Methods}

\subsection{Materials}

Açaí powder and polydextrose were purchased from
Sun Foods Inc. (Campinas, Brazil) and Danisco Inc. (São Paulo, Brazil). Wheat flour, sugar, salt, hydrogenated vegetable fat, compressed yeast and water were purchased from local markets in Fortaleza.

\subsection{Methods}

\subsubsection{Preparation of Breads}

The control bread was composed by $300.0 \mathrm{~g}$ of wheat flour, $120.0 \mathrm{~g}$ water, $30.0 \mathrm{~g}$ hydrogenate vegetable fat, $15.0 \mathrm{~g}$ refined sugar, $11.0 \mathrm{~g}$ dry yeast, $2.0 \mathrm{~g}$ salt and the inclusion of açaí powder and polydextrose was based on the Central Composite Rotational Design (CCRD) as shown in Table 1.

All ingredients were mixed in a semi-industrial mixer (Lieme BP06, Brazil), following a preliminary mixing of dry ingredients for $1 \mathrm{~min}$ at low speed. Mixing was done for 3 minutes at medium speed and for 6 minutes at high speed. A quantify of water was added in the mixer at the beginning of the medium speed period. Dough temperature at the end of mixing was $T=26.0^{\circ} \mathrm{C} \pm 1.0^{\circ} \mathrm{C}$. After the mixture the dough was left to rest for $5 \mathrm{~min}$ and then divided in pieces of $100.0 \mathrm{~g}$ and hand-molded in an ellipses form. Dough fermentation was performed in fermentation chamber (Perfecta, Brazil) at $28.0{ }^{\circ} \mathrm{C} \pm 2.0{ }^{\circ} \mathrm{C}$ and $70.0 \%$ relative humidity for two hours in fermentation process. The doughs were baked without stream at $220.0{ }^{\circ} \mathrm{C}$ for 20 minutes and cooled at room temperature $\left(28.0{ }^{\circ} \mathrm{C} \pm 2{ }^{\circ} \mathrm{C}\right)$.

Table 1 Second order design matrix used for the evaluation of the effects of ingredients addition variables (açaí powder and polydextrose) on some physical, structural and sensory properties of breads.

\begin{tabular}{lllll}
\hline & \multicolumn{2}{c}{ Coded values } & \multicolumn{2}{c}{ Uncoded values } \\
\cline { 2 - 5 } Runs & Açaí powder $(\mathrm{g} / 100 \mathrm{~g}) \mathrm{X}_{1}$ & Poly dextrose $(\mathrm{g} / 100 \mathrm{~g}) \mathrm{X}_{2}$ & Açaí powder $(\mathrm{g} / 100 \mathrm{~g}) \mathrm{X}_{1}$ & Poly dextrose $(\mathrm{g} / 100 \mathrm{~g}) \mathrm{X}_{2}$ \\
\hline 1 & -1 & -1 & 15 & 15 \\
2 & -1 & +1 & 15 & 45 \\
3 & +1 & -1 & 45 & 15 \\
4 & +1 & +1 & 45 & 45 \\
5 & 0 & -1.41 & 30 & 0 \\
6 & 0 & +1.41 & 30 & 60 \\
7 & -1.41 & 0 & 0 & 30 \\
8 & +1.41 & 0 & 60 & 30 \\
9 & 0 & 0 & 30 & 30 \\
10 & 0 & 0 & 30 & 30 \\
11 & 0 & 0 & 30 & 30 \\
\hline
\end{tabular}




\subsubsection{Analyses of Breads}

Physical properties of bread. Bake loss of breads was determined according to:

$$
\operatorname{Bake\operatorname {Loss}}(\%)=\left[W_{b b}-\left(\frac{W_{a b}}{W_{b b}}\right)\right] \times 100
$$

where $\mathrm{W}_{\mathrm{bb}}$ is the weight of the loaf before baking and $\mathrm{W}_{\mathrm{ab}}$ is the weight of the loaf after baking and cooling [17].

\section{(1) Specific Volume}

Weight and loaf volume after baking were evaluated. The specific loaf volume was calculated by dividing loaf volume and weight (method 10-10B) [18]. Specific volume was expressed in $\mathrm{cm}^{3} / \mathrm{g}$.

(2) Texture of the Bread Crumbs

Bread firmness was measured as described in AACC Method 74-09 [18] using a TA.XT2 instrument (Texture Technologies, New York, NY, USA) with a $40 \mathrm{~mm}$ diameter cylindrical probe, for $25 \%$ of compression, at a test speed of $1.0 \mathrm{~mm} / \mathrm{min}$. At least four slices of bread ( $25 \mathrm{~mm}$ thickness each) cut from the center of the loaf were tested.

(3) Color Analysis

The crust and crumb colors were measured using Hunter Lab Color Flex (Hunter Associates Inc., Reston, VA, USA). Averages of three measurements of $\mathrm{L}^{*}, \mathrm{a}^{*}$ and $\mathrm{b}^{*}$ values were recorded. Color measurements of bread crumb (20 measurements/runs) were performed. Results were expressed as browning index (BI) according to the following equations [18, 19].

$$
\begin{gathered}
\qquad X=\frac{a *+1.75 L^{*}}{5.645 L^{*}+a^{*}-3.012 b^{*}} \\
\text { Browning Index }(B I)=\frac{100(X-0.31)}{0.172}
\end{gathered}
$$

Image analysis. The bread was sliced transversely with a slicing machine. The images were captured using a color camera (NIKON P610, Nikon Inc., Japan) with a resolution of $4,272 \times 2,848$ pixels. The camera was located at $20 \mathrm{~cm}$ overhead on the sample platform to obtain the best image of the samples of bread slices throughout the experiment. The samples were illuminated with two $36 \mathrm{~W}$ fluorescent lights (model: T8 G13, Phillips, Brazil) and enclosed in a black cardboard box with a door [20]. The inside wall of the box was concealed with black paper to reduce the reflectance, and the roof was covered with white paper to enhance light scattering and reduce the shadow [21]. The sliced bread samples were positioned on a black background to provide higher contrast between the background and the sample. The camera was connected to a computer, which contained remote shooting software Nikon Wireless Mobile Utility (WMU) (version 1.5.0, Nikon INC, Japan) through which the digital images were acquired.

The digital images were made using ImageJ software (National Institute of Health, Bethesda, MD, USA). The image was split into color channels. The contrast was enhanced and, finally, the image was binarized after applying a grey scale threshold. This was performed with the aim of dividing images into regions of cells and surrounding cell wall material. The analysis was performed on a slice area of $15 \times 17$ $\mathrm{mm}$. Porosity measurements were measured in triplicate [22].

(1) Scanning Electron Microscopy (SEM) Images of Breads

Inspect F50 Scanning Electron Microscope (FEI, Czech Republic) was used to examine flour and crumb structures. Bread crumbs were cut into cubes $\left(1 \mathrm{~cm}^{3}\right)$. An accelerating voltage of $7 \mathrm{kV}$ and $10 \mathrm{kV}$ was used for flours and breads; system pressures were $80 \mathrm{~Pa}$ and $110 \mathrm{~Pa}$ for flours and breads, respectively.

(2) Statistical Analysis and Modeling

The CCRD was performed to obtain a second order model to predict the quality of dough and bread as a function of açaí powder and polydextrose addition. This model can be observed in the following equation:

$$
y=\beta_{0}+\sum_{i<j} \beta_{i j} x_{i} x_{j}+\sum_{j} \beta_{j j} x_{j}^{2}+\varepsilon
$$

where $y$ is the predicted response (dough and bread quality variables), $\beta_{0}$ is the global mean, $\beta_{\mathrm{j}}$ is the linear coefficient, $\beta_{\mathrm{ij}}$ is the coefficient of interaction, $\beta_{\mathrm{jj}}$ is the quadratic coefficient, $\varepsilon$ is the error of the model, and $x_{\mathrm{i}}$ 
and $x_{\mathrm{j}}$ are the coded values of the independent variables.

The experimental data were analyzed using Statistica software, version 8.0 (Statsoft, Inc., Tulsa, OK, USA). Analysis of variance (ANOVA) tables was generated, and the effect and regression coefficients of individual linear, quadratic and interaction terms were determined. The significances of all words were judged statistically according to the $p$-value, which was set at a 5\% significance level. The quality-of-fit of the model equation was expressed by the coefficient of determination $\left(\mathrm{R}^{2}\right)$, and its statistical significance was determined using the F-test. For validation of the statistical results, the observed values of dough and bread quality variables were compared with the predicted values obtained by the experimental models. The independent variables optimized were $X_{1}$ (Açaí Powder-AP), $\mathrm{X}_{2}$ (Polydextrose-PO) for dependent response $\mathrm{Y}_{1}$ (Bake Loss), $\mathrm{Y}_{2}$ (Specific Volume), $\quad \mathrm{Y}_{3}$ (Alveoli Number), $\mathrm{Y}_{4} \quad\left(\mathrm{~L}^{*}\right), \mathrm{Y}_{5}$ (Browning index) and $\mathrm{Y}_{6}$ (Hardness). The complete design consisted of 11 combinations performed in standard order (Table 1).

\section{Results and Discussion}

Quality parameters of bread enriched with açaí powder and polydextrose are tabulated in Table 2. CCRD design of response surface methodology was chosen to show the main effects of independent variables (açaí powder and polydextrose) and their interactions. The bake loss, specific volume and alveoli number decreased significantly with increasing açaí powder and polydextrose level $(p<0.05$, Table 2).

The highest bake loss was determined to be $17.00 \%$ in the bread containing $30 \mathrm{~g} / 100 \mathrm{~g}$ of açaí powder and without addition of polydextrose, while the lowest to be $4.66 \%$ in the sample containing $45 \mathrm{~g} / 100 \mathrm{~g}$ of açaí powder and polydextrose. The inclusion of açaí powder and polydextrose in bread formulations significantly affected the specific and alveoli number. It produces more dense breads by reducing the volume and crumb porosity, characteristics of products enriched with fibers and minerals similarly reported by Martínez et al. [23].

There was a general trend indicating a decline in bread brightness with increasing açaí powder levels, but no significant difference $(p>0.05)$ to polydextrose levels. The browning index increased with açaí powder levels and decreased with increase polydextrose levels. This result was expected because the açaí powder presents low brightness and purple pigments [24]. And polydextrose consists of a white-cream powder, as reported by Almeida et al. [25] with high brightness.

Table 2 Quality properties of bread enriched with açaí powder and polydextrose.

\begin{tabular}{lllllll}
\hline \multirow{2}{*}{ Runs } & \multicolumn{5}{c}{ Quality properties } \\
\cline { 2 - 7 } & Bake loss $(\%)$ & Specific volume $\left(\mathrm{cm}^{3} / \mathrm{g}\right)$ & Alveoli number (cells) & $\mathrm{L}^{*}$ & Browning index & Hardness (N) \\
\hline 1 & $13.33 \pm 0.88$ & $3.33 \pm 0.17$ & $2,504 \pm 4$ & $62.70 \pm 0.05$ & $28.80 \pm 0.12$ & $2.30 \pm 0.03$ \\
2 & $8.33 \pm 0.33$ & $3.07 \pm 0.05$ & $1,341 \pm 40$ & $68.25 \pm 0.09$ & $24.30 \pm 0.10$ & $3.21 \pm 0.02$ \\
3 & $8.33 \pm 0.33$ & $2.07 \pm 0.04$ & $1,342 \pm 42$ & $52.40 \pm 0.05$ & $44.46 \pm 0.09$ & $3.59 \pm 0.02$ \\
4 & $4.66 \pm 0.33$ & $1.72 \pm 0.07$ & $1,041 \pm 7$ & $50.09 \pm 0.08$ & $48.44 \pm 0.08$ & $4.21 \pm 0.02$ \\
5 & $17.00 \pm 0.57$ & $2.83 \pm 0.06$ & $2,943 \pm 7$ & $58.18 \pm 0.05$ & $54.21 \pm 0.03$ & $2.05 \pm 0.03$ \\
6 & $3.66 \pm 0.33$ & $2.46 \pm 0.06$ & $829 \pm 23$ & $61.70 \pm 0.09$ & $27.91 \pm 0.04$ & $4.98 \pm 0.04$ \\
7 & $14.66 \pm 0.33$ & $3.10 \pm 0.04$ & $3,220 \pm 21$ & $72.19 \pm 0.04$ & $22.14 \pm 0.04$ & $3.06 \pm 0.03$ \\
8 & $8.33 \pm 0.33$ & $1.58 \pm 0.04$ & $888 \pm 18$ & $44.18 \pm 0.09$ & $69.34 \pm 0.04$ & $5.19 \pm 0.03$ \\
9 & $6.33 \pm 0.33$ & $2.35 \pm 0.05$ & $1,326 \pm 11$ & $52.10 \pm 0.08$ & $59.07 \pm 0.04$ & $3.59 \pm 0.02$ \\
10 & $6.33 \pm 0.33$ & $2.34 \pm 0.02$ & $1,334 \pm 4$ & $52.15 \pm 0.05$ & $59.20 \pm 0.03$ & $3.57 \pm 0.03$ \\
11 & $6.33 \pm 0.33$ & $2.33 \pm 0.02$ & $1,341 \pm 2$ & $52.16 \pm 0.03$ & $59.15 \pm 0.05$ & $3.58 \pm 0.02$ \\
\hline
\end{tabular}


The açaí powder and polydextrose in their linear models significantly influenced all quality attributes evaluated (Table 3), however, there was a greater quadratic effect of açaí powder than that of polydextrose, probably due to the high content of fibers, pigments and minerals and their contribution for gluten network modifications.

As summarized in Table 3, breads were prepared with açaí powder and polydextrose presented significant effects on the modifications of the expansion properties. It has been extensively reported that the addition of non-gluten ingredients reduced loaf volume of the bread, which was mainly attributed to the dilution of the gluten in formula, as well as the change of viscoelastic properties in the dough [26].

However, our study found that the inclusion of the ingredients is satisfactory at levels below $15 \mathrm{~g} / 100 \mathrm{~g}$ to açaí powder and $45 \mathrm{~g} / 100 \mathrm{~g}$ of polydextrose (Fig. 1). Specific volume is an important parameter to analyze the bread quality which involves bread volume/weight and together with bake loss represent the breadmaking quality set after the baking of bread. The specific volume of the breads ranged from $1.72 \mathrm{~cm}^{3} / \mathrm{g}$ to 3.33 $\mathrm{cm}^{3} / \mathrm{g}$, similar and inclusive higher values than other breads $\left(1.23-3.5 \mathrm{~cm}^{3} / \mathrm{g}\right)$ [27, 28].

The greater bread weight loss was observed for small additions of the ingredients. The minimum point was obtained for addition of polydextrose between 45 and $55 \mathrm{~g} / 100 \mathrm{~g}$ correlated with 35 to $45 \mathrm{~g} / 100 \mathrm{~g}$ of açaí powder. The inclusion of these ingredients probably caused the reduction in free water amount present in the dough available for the evaporation process to be attached to these components, reducing their loss in the baking process. This result can be explained by the action of polydextrose as soluble fiber, with potential for water absorption, and also by the dietary fibers present in the açaí $[29,30]$. Therefore, the use of these ingredients can increase the yield of the process.

Effect of açaí powder and polydextrose levels on bread hardness is shown in Fig. 2. A significant ( $p<$ 0.05 ) increase in bread hardness was noted at the highest substitution level. In general, the bread hardness increased with increase of açaí powder and polydextrose levels. The reduction of free water content in bread may be related to this result. The reduction of the moisture content with the increase of the bread crumb hardness was reported by several authors [31-33]. This may be due to increased water absorption which leads to increased crumb softness. Similar results were found by $\mathrm{Fu}$ et al. [34, 35] when enriched breads by $2 \%-8 \%$ turmeric powder or 3\%-9\% lemon fiber.

The color of bread crumb is an important parameter to determine its acceptability. Therefore, the effects of açaí powder and polydextrose addition on the bread crumb color were significantly. The prepared açaí powder-polydextrose (50:50) powder showed a medium brightness value ( $\left.\mathrm{L}^{*}: 40.38\right)$ and a low intensity of yellow ( $\left.b^{*}: 10.11\right)$ and red (a*: 4.28) colors. Therefore, its use in different proportions promoted changes in the bread browning index, especially when the açaí powder is in greater quantity.

Table 3 Regression coefficients for the dependent variables models in terms of açaí powder and polydextrose linear models, interaction and quadratic models.

\begin{tabular}{llllllll}
\hline Response & Intercept & AP & PO & AP $\times$ PO & $(\mathrm{AP})^{2}$ & $(\mathrm{PO})^{2}$ & Lack of fit \\
\hline Specific volume & 2.458 & $-0.470^{*}$ & $-0.112^{*}$ & -0.089 & 0.056 & 0.024 & 0.689 \\
Alveoli number & $1,294.386$ & $-510.660^{*}$ & $-474.167^{*}$ & $215.500^{*}$ & $182.538^{*}$ & $140.497^{*}$ & 0.294 \\
Bake loss & 6.353 & $-1.776^{*}$ & $-2.945^{*}$ & 0.528 & $0.992^{*}$ & $1.292^{*}$ & 0.944 \\
Hardness & 3.434 & $0.546^{*}$ & $0.615^{*}$ & -0.072 & $0.143^{*}$ & -0.009 & 0.377 \\
L* & 53.302 & $-7.040^{*}$ & $0.850^{*}$ & $1.439^{*}$ & $1.873^{*}$ & $-1.965^{*}$ & 0.482 \\
Browning index & 52.921 & $11.183^{*}$ & $-4.426^{*}$ & 2.119 & $-2.960^{*}$ & $-4.130^{*}$ & 0.685 \\
\hline
\end{tabular}

* means that there are statistically significant regression coefficients $p \leq 0.05$. 


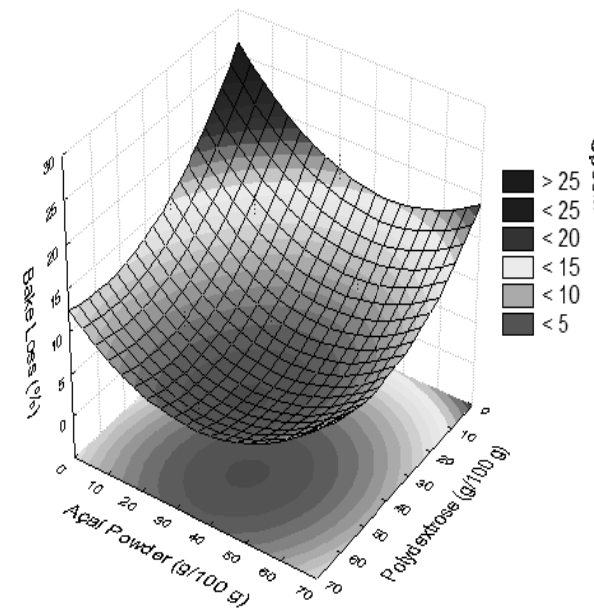

(a)

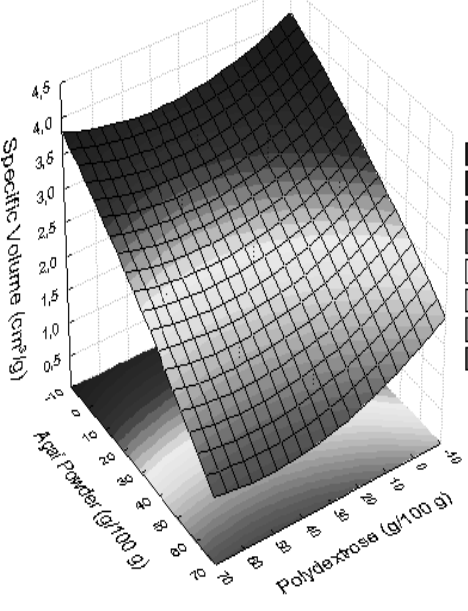

(b)
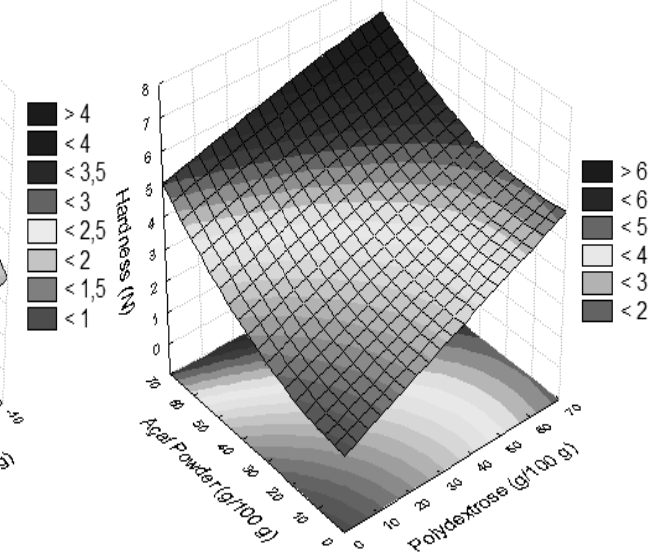

(c)

Fig. 1 3D surface response plots of bake loss (a), specific volume (b) and hardness (c).

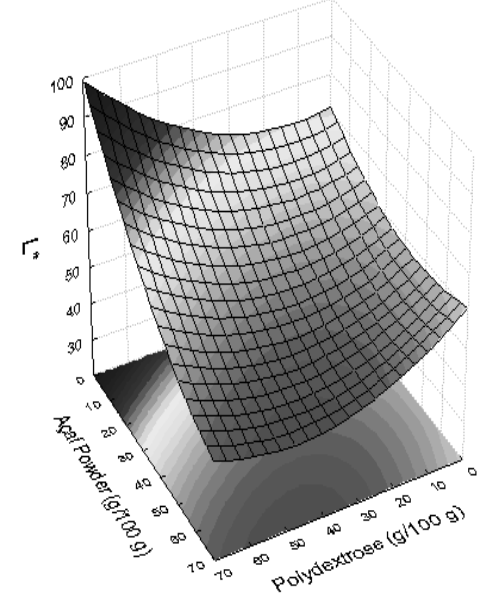

(a)

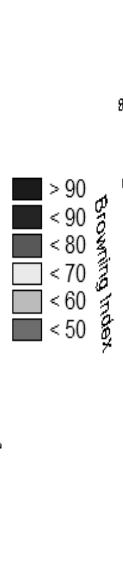

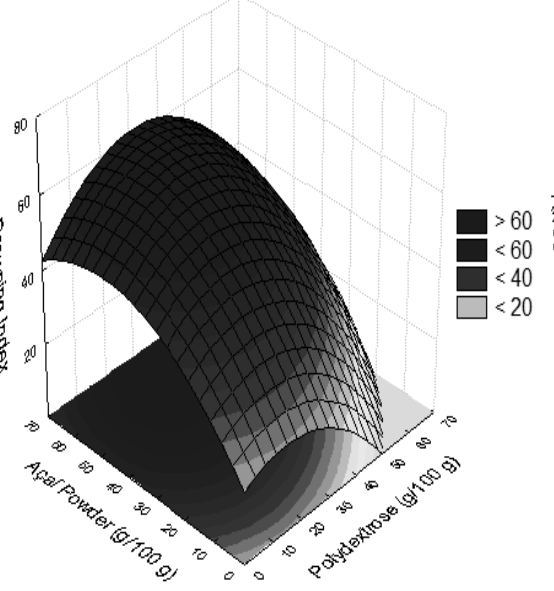

(b)

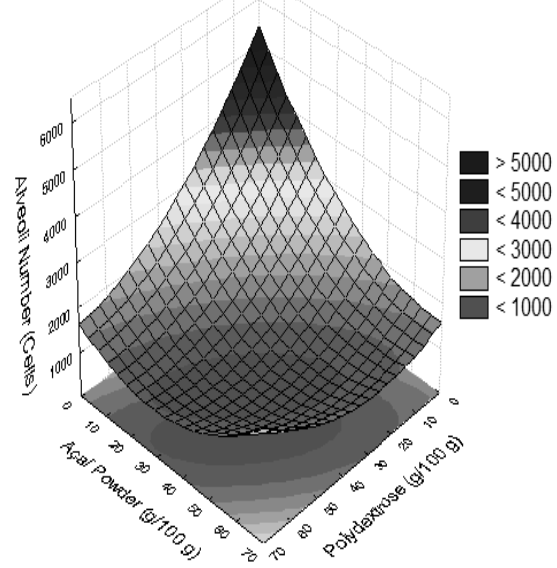

(c)

Fig. 2 3D surface response plots of $L^{*}(a)$, browning index (b) and alveoli number (c).

The physical quality of bread decreased significantly with addition of açaí powder and polydextrose. Numerous scientific reports have shown that partial replacing of the wheat flour with non-gluten products contributed to deterioration in bread quality, especially its volume [36-39].

Through electron microscopy, was possible to verify that the addition of powdered açaí and polydextrose in amounts up to $30 \mathrm{~g} / 100 \mathrm{~g}$ promoted permanent damages to the gluten network, reducing the quality of the bread through the specific volume and the alveoli number.

However, when the addition of açaí powder is 60 $\mathrm{g} / 100 \mathrm{~g}$, without the incorporation of polydextrose, a dehydration process of the starch molecules could be verified, most likely caused by the absorption of the free water by the açaí powder, suggesting a detriment in the interaction between the starch and gluten molecules.

According to Ref. [16], the functionality of the gluten network developed through mixing is crucial for gas retention and the final structure of bread. During proofing, the rate of gas production depends on the activity of baker's yeast (Saccharomyces cerevisiae) while the rate of dough expansion is determined by both the gas production and the rate of gas transfers. However, if the gluten network is not effective in capturing this gas, the expansion is compromised, as observed in this study by the incorporation of açaí powder and polydextrose in 
bread formulations.

The inclusion of açaí powder and polydextrose in different levels affected significantly the number of alveoli and bread crumb structure. At high levels of ingredients incorporation ( $>30 \mathrm{~g} / 100 \mathrm{~g}$ ) there was a strong reduction in alveoli number of bread crumb, reducing the product quality. According to Ref. [40], the yeast generated carbon dioxide and expanded the bread volume. Gas cell stabilization and gas retention are of interest because they determine the crumb structure and the bread volume. Thus, the açaí powder and polydextrose addition at high levels may decrease the retention of carbon dioxide due to the cracks promoted by these ingredients in the gluten network, as can be seen by scanning electron microscopy in Fig. 3.

Higher gassing power which is associated with high pressure means more production of carbon dioxide in the dough system; thus, higher yeast activity during fermentation [41, 42]. In general, high yeast activity was exhibited when no açaí powder and polydextrose was present in the dough system.

Thus, the densification of these breads could be mainly attributed to the interactions between gluten and fibrous material. In fact, dietary fiber contained in açaí powder could alter the formation of a continuous gluten network and exhibit a destabilizing effect at the interfaces of gas cells of the dough [43].
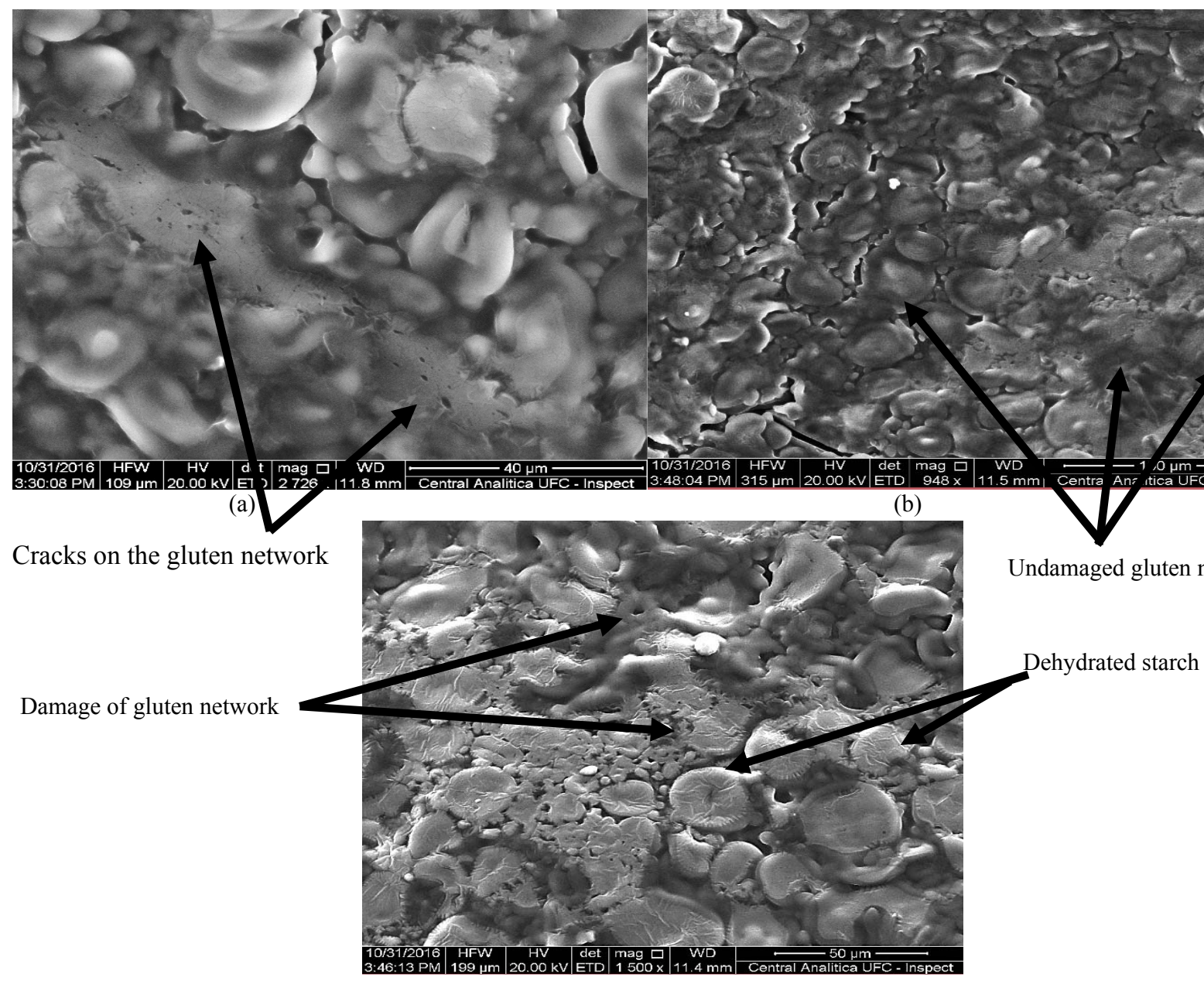

(c)

Fig. 3 Scanning electron microscopy of dough added with $45 \mathrm{~g} / 100 \mathrm{~g}$ of açaí powder and $45 \mathrm{~g} / 100 \mathrm{~g}$ of polydextrose (a), dough added with $30 \mathrm{~g} / 100 \mathrm{~g}$ of açaí powder and $30 \mathrm{~g} / 100 \mathrm{~g}$ of polydextrose (b) and dough added with $60 \mathrm{~g} / 100 \mathrm{~g}$ of açaí powder and $0 \mathrm{~g} / 100 \mathrm{~g}$ of polydextrose (c). 


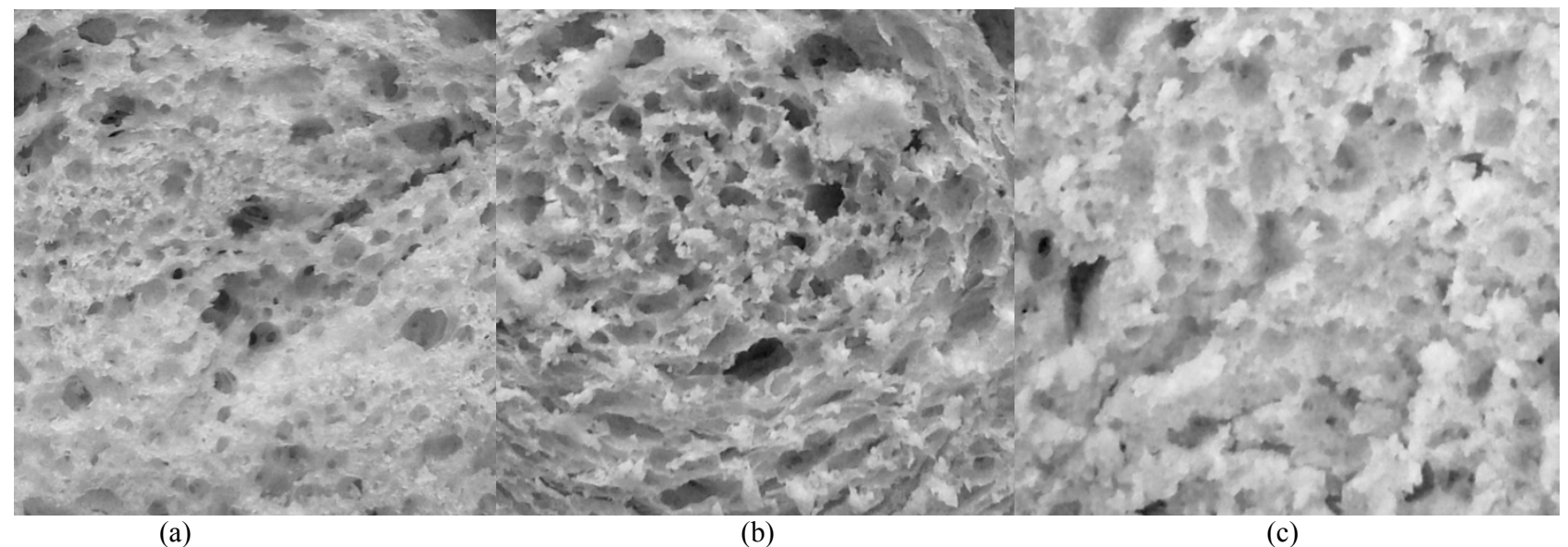

Fig. 4 Sample gray level images of $40 \times 40 \mathrm{~mm}$ field of view of bread crumb. Bread supplemented with $45 \mathrm{~g} / 100 \mathrm{~g}$ of açaí powder and $45 \mathrm{~g} / 100 \mathrm{~g}$ of polydexrose (a); Bread supplemented with $30 \mathrm{~g} / 100 \mathrm{~g}$ of açaí powder and $30 \mathrm{~g} / 100 \mathrm{~g}$ of polydexrose (b); Breadsupplemented with $65 \mathrm{~g} / 100 \mathrm{~g}$ of açaí powder and $0 \mathrm{~g} / 100 \mathrm{~g}$ of polydexrose (c).

The bread crumbs were analyzed by digital image (Fig. 4). Açaí powder and polydextrose alter the macroscopic bread crumb structural parameters.

In contrast, the addition of these ingredients in proportions of $30 \mathrm{~g} / 100 \mathrm{~g}$ increases the mean cell area and the number of cells $/ \mathrm{cm}^{2}$. Visual texture defines the appearance of the product, ranging from the color of the crust to the loaf volume to the cellular structure of the individual slices [44]. Visual texture accounts for up to $20 \%$ of the weighting score when assessing bread quality [45]. The reduction of the crumb porosity by the weakening of gluten through the addition of the açaí powder and polydextrose can affect the bread quality.

\section{Conclusions}

Açaí powder and polydextrose can be used in the production of breads. According to the optimization of results it could be concluded that the ingredients addition up to $30 \mathrm{~g} / 100 \mathrm{~g}$ can be used in the bread formulation without impairing quality characteristics.

\section{Acknowledgment}

The authors would like to thank the Central Analítica-UFC/CT-INFRA/MCTI-SISANO/Pró-Equi pamentos CAPES and Federal University of Ceará (UFC) for the support.

\section{References}

[1] Kutyła-Kupidura, E. M., Sikora, M., Krystyjan, M., Dobosz, A., Kowalski, S., and Pysz, M. 2015. "Properties of Sugar-Free Cookies with Xylitol, Sucrose, Cesulfame K., and Their Blends." Journal of Food Process Engineering 39: 321-9.

[2] Arts, I. C., and Hollman, P. C. 2005. "Polyphenols and Disease Risk in Epidemiologic Studies." The American Journal of Clinical Nutrition 81 (1): 317S-25S.

[3] Marchesi, J. R., Adams, D. H., Fava, F., Hermes, G. D., Hirschfield, G. M., and Hold, G. 2016. "The Gut Microbiota and Host Health: A New Clinical Frontier." Gut 65 (2): 330-9.

[4] Rogez, H. 2000. Açai: Preparo, Composicao e Melhoramento da Conservacao (first ed.), Brazil: EDUFPA.

[5] Moura, R. S., Ferreira, T. S., Lopes, A. A., Pires, K. M., Nesi, R. T., and Resende, A. C. 2012. "Effects of Euterpe Oleracea Mart. (ACAI) Extract in Acute Lung Inflammation Induced by Cigarette Smoke in the Mouse." Phytomedicine 19: 262-9.

[6] Auerbach, M. H., Craig, S. A., Howlett, J. F., and Hayes, K. C. 2007. "Caloric Availability of Polydextrose." Nutrition Reviews 65: 544-9.

[7] Colliopoulos, J. A., John, J. G., and Tsau, J. H. 1986. Sweetening Composition. United States Patent, 4631195.

[8] Mitchell, H., Auerbach, M. H., and Moppett, F. K. 2001. "Polydextrose L.O. Nabors (Ed.)." Alternative Sweeteners (3rd ed.) New York: Marcel Dekker, 499-518.

[9] Ibarra, A., Astbury, N. M., Olli, K., Alhoniemi, E., and Tiihonen, K. 2015. "Effects of Polydextrose on Different Levels of Energy Intake: A Systematic Review and Meta-Analysis." Appetite 87: 30-37. 
[10] Ibarra, A., Astbury, N. M., Olli, K., Alhoniemi, E., and Tiihonen, K. 2016. "Effect of Polydextrose on Subjective Feelings of Appetite during the Satiation and Satiety Periods: A Systematic Review and Meta-Analysis." Nutrients 8: 45.

[11] Aidoo, R. P., Afoakwa, E. O., and Dewettinck, K. 2014. "Optimization of Inulin and Polydextrose Mixtures as Sucrose Replacers during Sugar-Free Chocolate Manufacture-Rheological, Microstructure and Physical Quality Characteristics." Journal of Food Engineering 126: 35-42.

[12] Kasprzak, M. M., Laerke, H. N., and Knudsen, K. E. B. 2012. "Effects of Isolated and Complex Dietary Fiber Matrices in Breads on Carbohydrate Digestibility and Physicochemical Properties of Ileal Effluent from Pigs." Journal of Agricultural and Food Chemistry 60 (51): 12469-76.

[13] Kopec, A., Pysz, M., Borczak, B., Sikora, E., Rosell, C. M., and Collar, C. 2011. "Effects of Sourdough and Dietary Fibers on the Nutritional Quality of Breads Produced by Bake-Off Technology." Journal of Cereal Science 54 (3): 499-505.

[14] Stojceska, V. 2011. 'Dietary Fiber from Brewer' s Spent Grain as a Functional Ingredient in Bread Making Technology." In Flour and Breads and Their Fortification in Health and Disease Prevention 171-81.

[15] Arendt, E. K., and Zannini, E. 2013. "Wheat and Other Triticum Grains." In Cereal Grains for the Food and Beverage Industries. United Kingdom: Wood Head Publishing, 1-67.

[16] Gao, J., Koh, A. H. S., Tay, S. L., and Zhou, W. 2017. "Dough and Bread Made from High and Low Protein Flours by Vacuum Mixing: Part 1: Gluten Network Formation." Journal of Cereal Science 74.

[17] Alvarez-Jubete, L., Auty, M., Arendt, E. K., and Gallagher, E. 2010. "Baking Properties and Microstructure of Pseudocereal Flours in Gluten-Free Bread Formulations." Eur. Food Res. Technol. 230: $437-45$.

[18] AACC. 2000 American Association of Cereal Chemists Approved Methods of the AACC (tenth ed.), St. Paul, MN: EUA.

[19] Buera, M. P., Retriella, C., and Lozano, R. D. 1985. "Definition of Color in the Non-enzymatic Browning." Die Farbe 33: 316-26.

[20] Saricoban, C., and Yilmaz, M. T. 2010. "Modelling the Effects of Processing Factors on the Changes in Colour Parameters of Cooked Meatballs Using Response Surface Methodology." World Applied Sciences Journal 9 (1): 14-22.

[21] Al-Rahbi, S., Manickavasagan, A., Al-Yahyai, R., Khriji, L., and Alakahakoon, P. 2013. "Detecting Surface Cracks on Dates Using Color Imaging Technique.” Food Science and Technology Research 19: 795-804.

[22] Al-Ohali, Y. 2011. "Computer Vision Based Date Fruit Grading System: Design and Implementation.” Journal of King Saud University-Computer and Information Sciences 23: 29-36.

[23] Martínez, M. M., Román, L., and Gómez, M. 2018. "Implications of Hydration Depletion in the Vitro Starch Digestibility of White Bread Crumb and Crust." Food Chemistry 239: 295-303.

[24] Huang, G., Guo, Q., Wang, C., Ding, H. H., and Cui, S. W. 2016. "Fenugreek Fiber in Bread: Effects on Dough Development and Bread Quality." LWT-Food Science and Technology 71: 274-80.

[25] Efraim, P., Silva, L. B., Queiroz, M. B., Fadini, A. L., Fonseca, R. C. C., and Germer, S. P. M. 2016. "Chewy Candy as a Model System to Study the Influence of Polyols Nd Fruit Pulp (AçaÍ) on Texture and Sensorial Properties." LWT-Food Science and Technology 65: 268-74.

[26] Almeida, E. L., Chang, Y. K., and Steel, C. J. 2013. "Dietary Fiber Sources in Bread: Influence on Technological Quality." LWT_Food Science and Technology 50 (2): 545-53.

[27] Brites, C., Trigo, M. J., Santos, C., Collar, C., and Rosell, C. M. 2010. "Maize-Based Gluten-Free Bread: Influence of Processing Parameters on Sensory and Instrumental Quality." Food Bioprocess Technology 3: 707-15.

[28] Wieser, H., and Koehler, P. 2012. "Detoxification of Gluten by Means of Enzymatic Treatment." Journal of AOAC International 95: 356-63.

[29] Saura-Calixto, F., Rufino, M. S. M., Jiménez, J., Arranz, S., Alvez, R. E., Brito, E. S., and Oliveira, M. S. P. 2011. "AçaÍ (Euterpe Oleraceae) 'BRS ParÁ': A Tropical Fruit Source of Antioxidant Dietary Fiber and High Antioxidant Capacity Oil." Food Research International 44: 2100-6.

[30] Blaak, E. E., Konings, E., Schoffelen, P. F., and Stegen, J. 2014. "Effect of Polydextrose and Soluble Maize Fiber on Energy Metabolism, Metabolic Profile and Appetite Control in Overweight Men and Women, British." Journal of Nutrition 111: 111-21.

[31] He, H., and Hoseney, R. C. 1990. "Changes in Bread Firmness and Moisture during Long-Term Storage." Cereal Chemistry 67: 603-5.

[32] Besbes, E., Jury, V., Monteau, J., and Le-Bail, A. 2014. "Effect of Baking Conditions and Storage with Crust on the Moisture Profile, Local Textural Properties and Staling Kinetics of Pan Bread." LWT-Food Science and Technology 58: 658-66.

[33] Mudgil, D., Barak, S., and Khatkar, S. 2016. "Optimization of Bread Firmness, Specific Loaf Volume 
and Sensory Acceptability of Bread with Soluble Fiber and Different Water Levels." Journal of Cereal Science 70: 186-91.

[34] Fu, J. T., Chang, Y. H., and Shiau, S. Y. 2015. "Rheological, Antioxidative and Sensory Properties of Dough and Mantou (Steamed Bread) Enriched with Lemon Fiber." LWT- Food Science and Technology 61: 56-62.

[35] Lim, S., Park, S. H., Ghafoor, K., Hwang, S. Y., and Park, J. 2011. "Quality and Antioxidant Property of Bread Containing Turmeric (Curcuma longa L.) Cultivated in South Korea." Food Chemistry 112: 157-82.

[36] Matuda, T. G., Chevallier, S., Filho, P. A. P., Le-Bail, A., and Tadini, C. C. 2008. "Impact of Guar and Xanthan Gums on Proofing and Calorimetric Parameters of Frozen Bread Dough.” Journal of Cereal Science 48: 741-6.

[37] Simsek, S., Ohm, J., and Khalid, K. H. 2017. "Whole Wheat Bread: Effect of Bran Fractions on Dough and End-Product Quality." Journal of Cereal Science.

[38] Zouari, N., Fakhfakh, N., Jdir, H., Jridi, M., Rateb, M., Belbahri, L., Ayadi, M. A. A., and Nasri, M. 2017. "The Mallow, Malvaaegyptiaca L. (Malvaceae): Phytochemistry Analysis and Effects on Wheat Dough Performance and Bread Quality." LWT_Food Science and Technology 75: 656-62.

[39] Blandino, M., Locatelli, M., Gazzola, A., Coïsson, J. D., Giacosa, S., Travaglia, F., Bordiga, M., Reyneri, A.,
Rolle, L., and Arlorio, M. 2015. "Hull-Less Barley Pearling Fractions: Nutritional Properties and Their Effect on the Nutritional and Technological Quality in Bread-Making." J. Cereal Sci. 65: 48-56.

[40] Collar, C., and Angioloni, A. 2014. "Nutritional and Functional Performance of High $\beta$-Glucan Barley Flours in Breadmaking: Mixed Breads Versus Wheat Breads." Eur. Food Res. Technol. 238: 459-69.

[41] Jacobs, M. S., Izydorczyk, M. S., Preston, K. R., and Dexter, J. E. 2008. "Evaluation of Baking Procedures for Incorporation of Barley Roller Milling Fractions Containing High Levels of Dietary Fiber into Bread." $J$. Sci. Food Agric. 88: 558-68.

[42] Noort, M. W. J., Van Haaster, D., Hemery, Y., Schols, H. A., and Hamer, R. J. 2010. "The Effect of Particle Size of Wheat Bran Fractions on Bread Quality_Evidence for Fiber-Protein Interactions." Journal of Cereal Science 52: 59-64.

[43] Cauvain, S. P., and Young, L. S. 2006. "Baked Products: Science, Technology and Practice." Wiley Online Library.

[44] Scanlon, M., and Zghal, M. 2001. "Bread Properties and Crumb Structure.” Food Research International 34 (10): 841-64.

[45] Bell, S., Wang, I. S., and Austin, P. 2011. "It's a Maze: The Pore Structure of Bread Crumbs." Journal of Cereal Science 54 (2): 203-10. 